\title{
Incipient motion of coarse particles under regular shoaling waves
}

(Manuscript submitted to Coastal Engineering, December 2004)

Revised and resubmitted, June 2005

Terrile, Emanuele

Delft University of Technology, The Netherlands

University of Genoa, Italy

Terrile@diam.unige.it

Reniers, Ad J.H.M.

Delft University of Technology, The Netherlands

Stive, Marcel J.F.

Delft University of Technology, The Netherlands

M.J.F.Stive@citg.tudelft.nl

(corresponding author)

Tromp, Maarten

Delft University of Technology, The Netherlands

Verhagen, Henk Jan

Delft University of Technology, The Netherlands

H.J.Verhagen@citg.tudelft.nl 


\begin{abstract}
Incipient motion of coarse particles under regular shoaling waves is studied. Experiments are performed to investigate the effects of bed fluid acceleration on coarse particle stability. By varying wave height, wave period and water depth combinations of similar peak orbital velocities and weak to strong intra-wave accelerations were created. The particles used in these experiments have two different sizes both of a $\mathrm{cm}$ order-of-magnitude. The data confirm that acceleration plays a role for the initiation of motion, since combinations of similar orbital velocity and varying acceleration magnitude resulted in no motion, some motion and motion as acceleration increased. Qualitatively we found that initiation of motion occurs at or is very close to the maximum shear stress due to the combined effects of drag/lift and acceleration as introduced by Nielsen and Callaghan (2003). However, quantitatively their formulation does not lead to convincing discrimination between motion and no motion. We expect this to be due to the assumption that the coefficients for drag/lift and acceleration in their formulation are taken equal. From literature and from plotting our data against the Keulegan-Carpenter number we expect that the coefficients strongly vary caused by flow separation effects.
\end{abstract}

To arrive at a more convincing discrimination between motion and no-motion we introduced a new fluid acceleration descriptor for nonlinear shoaling waves. The combination of this descriptor with a Reynolds number $\mathrm{Re}_{\mathrm{g}}$ clearly delineates the regions with particle motion and without particle motion and has the potential to serve as a descriptor of the incipient motion of coarse particles under nonlinear regular waves.

Key words: incipient particle motion, skewed waves, wave acceleration, KC-number

\title{
Introduction
}

Coastal sediment transport researchers have been aware of the importance of fluid accelerations or the associated horizontal pressure gradients for a long time. Bagnold (1963) already recognised the potential importance of wave-induced accelerations in general. Madsen (1974) showed that horizontal pressure gradients associated with steep fronts of waves or bores might induce bulk instability and hence vastly enhanced shoreward sediment transport. Nielsen (1979) discussed the likely Keulegan-Carpenter number effects associated with accelerations in wave sediment transport. Hallermeier (1980) experimentally investigated initiation of motion by regular, symmetric waves for relatively coarse sand and showed that a Shields-like parameter could describe the discrimination between motion and no motion. An interesting experiment was done by King (1991) measuring different net sediment transport rates for forward facing and backward facing saw-tooth half-waves. The importance of surf zone waves often having saw-tooth asymmetry was discussed by Nielsen (1992), which leads to acceleration asymmetry and to thinner boundary layers ( greater shear stresses) associated with those peak velocities, which follow the briefest acceleration process. Sleath (1994) defined a quasi-steady regime and a pressure gradient-regime for coastal sediment transport separated by the value of an acceleration parameter, and in several subsequent works, e $\mathrm{g}$, Zala-Flores and Sleath (1998) and Sleath (1999) acceleration effects in wave induced sediment transport were quantified in terms of this parameter. The sheetflow data of Ribberink et al (2000) showed that real waves in a flume, as opposed to Stokes-wave like velocities in Utubes generated at least two times more sediment transport for the same orbital velocity magnitude, a difference which could be due to either saw tooth asymmetry or boundary layer streaming being present in the flume waves but not in the U-tube experiments. The discussion of Nielsen \& Callaghan (2003) subsequently provided quantitative estimates of the relative importance of streaming versus acceleration asymmetry. Drake and Calantoni (2001) made a 
quantitative process-based model to incorporate the effect of pressure gradients. Hoefel and Elgar (2003) used their results to show that flow acceleration may play a role in predicting onshore bar movement under moderate wave conditions. Although the effect of flow acceleration for fine particles is not well established yet, the above findings inspired our research group to undertake a series of experiments to further explore the role of flow acceleration. Since the effect is stronger for coarser particles (larger spatial pressure gradients) we decided to undertake experiments for unsteady flow with coarse particles of $(\mathrm{O}) \mathrm{cm}$ diameter. In the region just before wave breaking near bed fluid accelerations are found to be strong enough to move the coarse sediment. While the relevance for sand transport has yet to be established, the relevance for stability of bed protection and for onshore gravel transport clearly exists.

\section{Experimental set-up and method}

The experiments were carried out in a wave flume of the Laboratory of Fluid Mechanics of Delft University of Technology. The glass-walled flume has an effective length of $42.00 \mathrm{~m}$, a width of $0.80 \mathrm{~m}$ and a height of $1.00 \mathrm{~m}$. On the bottom of the flume a concrete slope was constructed with a gradient of 1:30 (Figure 1). Regular waves were generated with a wave paddle using second order wave steering and measurements were confined to the shoaling region. In order to compensate the influence of re-reflecting waves in the flume, an Active Reflection Compensation system was used. Surface elevations were measured with six resistance type wave gauges (Figure 1). Measurements confirmed that reflection coefficients on the 1 in 30 slope were low, viz. $1,2 \%$ on average.

Fluid motions were measured with an EMS (Electro Magnetic Flow Sensor) positioned about $5 \mathrm{~cm}$ above the bottom at various locations along the slope. Intra-wave variation of the orbital velocities and accelerations were derived by ensemble-averaging over the wave phase (records between 30-50 seconds were used implying averaging over 10 to 16 waves).

Two types of nearly uniform coarse particles $\left(\mathrm{D}_{90} / \mathrm{D}_{10} \cong 1.25\right)$ were applied in the experiments: $\mathrm{D}_{50}=8.8 \mathrm{~mm}$ and $\mathrm{D}_{50}=11.4 \mathrm{~mm}$. The specific density of the particles was $2.67 \mathrm{~kg} / \mathrm{m}^{3}$. In the shoaling region 5 strips of coloured particles (different colours per strip) were placed that could move, while outside these strips the particles were fixed to the bottom. For each test performed the number of particles were determined that moved by observing the measuring area at a particular strip, and the associated forcing wave flow properties, viz. the intra-wave near-bed velocity and acceleration variations. The tests were carried out with different wave periods in the range between $\mathrm{T}=2 \mathrm{~s}$ and $\mathrm{T}=4.4 \mathrm{~s}$, with different wave heights: $0.125,0.15$, $0.175,0.20 \mathrm{~m}$. and at two water depths $\mathrm{h}=0.60$ and $0.65 \mathrm{~m}$ respectively.

In order to create a statistically significant dataset a sufficient number of forcing situations is needed to establish the threshold of coarse particle motion, i.e. we need a sufficient number of situations with similar orbital velocity magnitudes in combination with small to large acceleration magnitudes. Some of these combinations did not lead to particle movement while others with similar orbital velocities, but larger acceleration did lead to particle movement. To establish a reliable percentage of particle motion, tests with near-equal velocities and accelerations for which particle motion occurred were repeated multiple times (up to 6 or 7 times). In doing so we were able to reduce stochastic effects, for instance due to insufficient settling time of the bed after placing which can result in a motion-favourable position for a particle. A total of 122 experimental results were established covering a range of orbital velocities and orbital accelerations. 
As indicated the stochastic nature of the particle movement needs consideration, therefore we have categorized the particle movement in classes (Table 1). Threshold of motion is defined as movement of at least 3 coloured particles out of a coloured strip. Movement of particles within a strip is not considered as movement. The percentages refer to the ratio of the times that three stones moved over the total of times that particle movement was assessed under near-equal conditions.

\begin{tabular}{|c|c|}
\hline $\begin{array}{c}\text { Particle movement } \\
\text { percentage }\end{array}$ & Classification \\
\hline less than $25 \%$ & never \\
between $25 \%$ and $75 \%$ & sometimes \\
more than $75 \%$ & always \\
\hline
\end{tabular}

Table 1: Classes of stone movements.

Using movement of at least 3 particles reduces the influence of the orientation of a particle in the bed. A sensitivity analysis, in which the data of the motion of 1 and 4 particles were also analysed, shows small differences with the results found for the chosen criterion of movement of 3 particles.

The above approach results in a dataset, which determines no, some, or significant particle movement with associated peak orbital motion and a representative measure of acceleration. This dataset forms the basis of our analysis presented below.

\section{Data analysis}

As a first step in our data analysis we compare our results with the classical findings of Hallermeier (1980). As far as we are aware this author was the first to undertake experiments for initiation of sediment motion under regular, non-shoaling waves. By analysing his data Hallermeier showed that the following relation produced a good discrimination between motion and no-motion.

$$
\frac{A}{D}=\left(\frac{8(s-1) g}{\omega^{2} D}\right)^{0.5}
$$

where $A$ is the wave orbital amplitude, $D$ the diameter of the spherical grains, $s$ is the relative density $\left(\rho_{s} / \rho\right), g$ the acceleration of gravity, and $\omega$ is the angular frequency $(2 \pi / T)$. If following Nielsen (1992) we define a Shields-like parameter as follows:

$$
\theta=\frac{0.5 f(A \omega)^{2}}{(s-1) g D}
$$

where $f$ is a wave friction factor, it can be shown that Hallermeier's expression can be rewritten to yield:

$$
\theta=4 f
$$

Hence Hallermeier's equation 1 is basically a Shields-like parameter, which expresses the ratio of drag/lift force over gravity force. Figure 2 displays our results versus Hallermeier's empirical relation. We note two important findings. Firstly, there appears to be no 
discrimination between our results for motion and no motion. This is obviously due to the fact that no acceleration effects are included in Hallermeier's parameter. Secondly, an interesting qualitative agreement with our results and the empirical fit of Hallermeier is observed, realizing that our results are "around-initiation-of-motion". Hence, in the absence of discriminative acceleration effects Hallermeier's result could be quantitatively modified to fit with our results. The quantitative modification is most probably due to the fact that our results are for a different diameter regime. However, this is beyond our present objectives.

As a second step in our data analysis we discuss our visual and video observations. Figure 3 depicts a typical time evolution of the free stream orbital velocity and the associated accelerations. Initiation of motion was observed to occur consistently in between the moment of maximum acceleration (point B) and the moment of maximum onshore flow velocity (point C). Apparently, the combination in this region of high acceleration and high free stream velocity creates an optimum condition for sediment particle instability.

The above analysis can be made quantitative by making use of the formulation proposed by Nielsen (1992, 2002) and Nielsen and Callaghan (2003) for the Shields parameter in an unsteady turbulent flow:

$$
\theta_{a}(t)=\frac{\frac{1}{2} f_{2.5}}{(s-1) g D_{50}}\left(u_{\infty} \cos \varphi_{\tau}+\frac{1}{\omega_{p}} \sin \varphi_{\tau} \frac{d u_{\infty}}{d t}\right)^{2} \times \operatorname{sign}\left(u_{*}(t)\right)
$$

where $f_{2.5}$ is the wave friction factor (Jonsson, 1966; and Jonsson and Svendsen, 1976) corresponding to a bed roughness of $2.5 D_{50}$ (Nielsen, 1992), $u_{\infty}$ is the free-stream velocity, $\omega_{p}$ is the peak angular frequency $(2 \pi / T)$ and $\varphi_{\tau}$ is the phase shift between free stream velocity and bed shear stress at the peak frequency. Note that in contrast to Nielsen (1992) the square of the summed effect instead of a linear summation of shear velocity and acceleration is taken in order to get the correct representation of the sediment flux bursts in a turbulent flow. Also note that for both effects the same coefficient is used, which will be discussed below.

Equation 4 expresses both the effect of shear velocity (first term) and of acceleration (second term) on the Shields parameter $\theta_{a}$. Making use of our measurements and by adopting a value of $1 / 18 \pi$ for $\varphi_{\tau}$ (which we have quantified from video observations and which is in line with Fredsoe and Deigaard, 1992) we have computed a typical intra-wave variation of the three contributing terms (Figure 4). The solid line, containing the symbol o, represents the pure velocity contribution $\left(u_{\infty} \cos \varphi_{\tau}\right)^{2}$, the solid line, containing the symbol $\mathrm{x}$, represents the acceleration contribution $\left(1 / \omega_{p} \sin \varphi_{\tau} \mathrm{d} u_{\infty} / \mathrm{d} t\right)^{2}$, the solid line, containing the symbol $\Delta$, represents their cross-product $\left(u_{\infty} \cos \varphi_{\tau} 1 / \omega_{p} \sin \varphi_{\tau} \mathrm{d} u_{\infty} / \mathrm{d} t\right)$ and the dash dot line the total value of the stability parameter $\theta_{a}$.

All three contributions are positive and strong for the particular region between point B and the passage of the wave crest. The instant at which the sum of these three terms is maximum, corresponding to a maximum value of the Shields number, is marked by the three symbols $(\Delta)$, (x) and (o). This corresponds to the region between (point $\mathrm{B}$ ) and the maximum onshore free stream velocity (point $C$ ), and is equal or very close to the instant of motion initiation that we have observed from video.

Qualitatively these results strongly confirm Nielsen and Callaghan's (2003) formulation as given by Equation 4, i.e. we observe that the maximum value of their formulation correlates clearly with the instant of motion initiation. Following Shields we therefore introduce a 
particle Reynolds-like number, since it is clear that the maximum orbital velocity and the stone diameter play a role as well:

$$
\operatorname{Re}_{g}=\frac{u_{r m s} D_{50}}{v}
$$

where $v$ is the kinematic viscosity of the fluid and $u_{r m s}=\sqrt{\operatorname{Var}\left[u_{\infty}(t)\right]}$.

Figures 5 and 6 show Nielsen and Callaghan's (2003) formulation for our experiments against the particle Reynolds-like number, where in Figure 6 we have used $u_{\max }$ instead of $u_{r m s}$. As we observe there is no convincing discrimination, which is very disappointing because of the qualitative agreement concluded before. In order to judge the goodness-of-discrimination in this case and in following cases we have designed a method that is described in Appendix 1.

As a next step in our further exploration of the issue we decided to first look for a more convincing discrimination between motion and no-motion by presenting our data as a function of a representative acceleration value, since our dataset contains situations with a similar orbital flow velocity and varying acceleration. From the above findings we concluded that two acceleration values play a crucial role in the process of initiation of motion, viz. $a_{0}$ being the value of acceleration at the beginning of the growth of the boundary layer when the velocity is zero and changing to onshore flow (point $\mathrm{A}$ in Figure 3 ) and $a_{\max }$ being the maximum of the near-bed fluid acceleration (point B in Figure 3). Obviously $a_{\max }$ should be large, but when $a_{0}$ is large as well the waves are either very skewed (saw-tooth like) or highly non-linear in a horizontal sense (horizontally asymmetric) and in both cases both the shear velocity and the acceleration are large, the combination of which favours motion. Therefore when the product of these values is large we should expect the conditions most conducive for initiation of sediment particle motion due to the acceleration. We thus introduce a new dimensional acceleration descriptor for nonlinear waves defined as follows:

$$
a_{n l}=\sqrt{a_{\max } a_{0}}
$$

The following properties for $a_{n l}$ are noted. Its value always lies between the value of $a_{0}$ and $a_{\max }$; in case of skewed waves, the closer it is to $a_{\max }$ the skewer or the more horizontally asymmetric the waves are. For waves that are horizontally asymmetric only, $a_{0}$ and $a_{\max }$ could be either the accelerations at and right after the change to onshore or the accelerations just before and at the change to offshore flow. In both cases the initiation of motion should be similarly likely. For pure sinusoidal waves no difference between $a_{\max }$ and $a_{0}$ exists, hence $a_{\max }$ is $a_{0}$, so $a_{n l}$ equals $a_{0}$, denoting minimal acceleration effects (as is expected to be the case for Hallermeier's results).

All our experimental results are collected in Figure 7 where for each test the measured $a_{n l}$, made dimensionless by the acceleration of gravity, is plotted versus the particle Reynolds-like number $\mathrm{Re}_{\mathrm{g}}$. A more convincing division is observed between a region in which there is always movement and one where there is no movement. This is also observed in the goodnessof-discrimination analysis in Appendix 1. Figure 7 includes a threshold of motion region indicative for the separation between motion and no motion. Apparently, for a particular value of $R e_{g}$ we observe a critical value of $a_{n l}$. When $a_{n l} \geq a_{n l, c r i t}$ the particles start to move.

Note that this particle Reynolds-like number increases with $D_{50}$ when $u_{r m s}$ is constant. If we expect the acceleration to play a role, initiation of motion should occur for larger values of $a_{n l}$, which is what we observe. On the other hand the number increases with $u_{r m s}$ while $D_{50}$ is 
constant. Apparently, even when the orbital velocity increases, we need an associated increase of acceleration, such that initiation of motion occurs only for larger values of $a_{n l}$. Initially we found this result difficult to interpret physically.

To extend our analysis we chose to plot (see Figure 8) $a_{n} / g$ versus $u_{r m s}{ }^{2} /\left(g D_{50}\right)$, which represent the two "Morrison-like" theoretical forces (drag/lift and pressure gradient) that cause the motion. We expected a linear decrease of the threshold motion line expressing compensation of the one force by the other, but although the tendency is not as clear as in Figure 7 the initiation of motion line is not decreasing but slightly increasing. Both these results can only be explained when the $C_{d}$ coefficient (the combined drag coefficient) decreases stronger than the $C_{m}$ coefficient (the acceleration coefficient) as $R e_{g}$ increases and a higher acceleration effect is required to compensate for the decrease in shear velocity effect. Looking at the results of Keulegan and Carpenter (1958) and Sarpkaya (1976) this can be observed to be the case for a variety of experiments in ranges of the Reynolds like number similar to our experiments. These conclusions are confirmed by plotting our results against the Keulegan-Carpenter number in Figure 9, which indicates that apparently our results fall in the region where strong variations are encountered in the degree of flow separation which influences both drag and acceleration. This conclusion makes the use of the same coefficient for the drag/lift and acceleration effect in Equation 4 (both $C_{m}$ and $C_{d}$ are equal to $f_{2.5}$ ) a questionable assumption.

From analysis of similar data by Tromp (2004) it was suggested that there might be a difference of as much as a factor of 5 between $C_{m}$ and $C_{d}$, viz. $C_{d} \approx 0.2 C_{m}$. Hence we applied this finding to equation 4. The results given in Figure 10 indicate that indeed a difference in discrimination is found locally, i.e. local improvement in the lower Re region, but overall the goodness-of-discrimination is similar to the original formulation (Appendix 1). Obviously one could make an empirical fit using a variable ratio between $C_{d}$ and $C_{m}$, but we consider this not justified on the basis of this limited dataset.

\section{Discussion}

In this discussion we address the question whether the new acceleration descriptor $a_{n l}$ has advantages compared to the acceleration descriptor $a_{\text {spike }}$ introduced by Drake and Calantoni (2001). To quantify sediment transport flux due to waves that are horizontally asymmetric and skewed they introduced the following formulation:

$$
\langle q\rangle=\left\{\begin{array}{cc}
k\left\langle u^{3}\right\rangle+K_{a}\left(a_{\text {spike }}-a_{\text {crit }}\right) & a_{\text {spike }} \geq a_{\text {crit }} \\
k\left\langle u^{3}\right\rangle & a_{\text {spike }}<a_{\text {crit }}
\end{array}\right.
$$

where $k$ and $K_{a}$ are empirical parameters, $a_{c r i t}$ is the critical value of $a_{\text {spike }}$ that must be exceeded before acceleration increases transport and $\left\langle u^{3}\right\rangle$ the average of the cubic of the velocity. Due to the definition of $\left.a_{\text {spike }}\left(=<a^{3}\right\rangle\left|<a^{2}\right\rangle\right)$ acceleration effects are absent for waves, which are horizontally asymmetric only. The effect of such waves is included in the average of the cubic of the velocity. In saw tooth waves the latter effect is absent and $a_{\text {spike }}$ represents the effect of acceleration in such waves.

Before commenting on these properties we explore the relation between $a_{n l}$ and $a_{\text {spike. }}$ We therefore simulated a range of different theoretical waveforms and compared the two descriptors. The time-varying velocity used in our simulations is: 


$$
u(t)=c \sum_{m=0}^{4} \frac{1}{2^{m}} \cos \left([m+1] \omega_{p} t+m \Phi\right)
$$

where $\Phi$ is the waveform parameter defined by Elgar and Guza (1985) and $c$ is a measure of the velocity amplitude.

We simulated five different waveform parameters, i.e. $\Phi=0, \pi / 8, \pi / 4,3 \pi / 8$ and $\pi / 2$, and five different values of $c=0.1,0.5,1.0,1.5$ and $2.0 \mathrm{~m} / \mathrm{s}$. In the following we only discuss results for horizontally asymmetric waves, i.e. $\Phi=0$, and for skewed waves, i.e. $\Phi=\pi / 2$, and compare the two descriptors $a_{n l}$ and $a_{\text {spike. }}$. In the first case, with $\Phi=0$ (Figure 11), $a_{\text {spike }}$ should be zero and $a_{n l}$ not. In fact, from Figure 12 we observe as expected that $a_{n l}^{3}$ behaves as $\left\langle u^{3}\right\rangle$. In the second case, with $\Phi=\pi / 2$ (Figure 13), the two descriptors behave similar and assume their maximum values, as expected. Figure 14 showing a linear proportionality between $a_{n l}$ and $a_{\text {spike }}$ confirms this. This linear proportionality also occurs for the waves in our experiments, because the waves are both skewed and horizontally asymmetric (Figure 15).

In conclusion we note that $a_{n l}$ includes both the effect of skewness and horizontal asymmetry. This simultaneous inclusion of skewness and horizontal asymmetry in one acceleration descriptor is attractive in the formulation of future bed load transport formulations.

\section{Conclusion}

Based on experiments with regular shoaling waves the effects of bed fluid acceleration on coarse particle stability were investigated for two different sizes both of a cm order-ofmagnitude. By varying wave height, wave period and water depth combinations of similar peak orbital velocities and weak to strong intra-wave accelerations were created. The data confirmed that the acceleration plays a role for the initiation of motion, since combinations of similar orbital velocity and varying acceleration magnitude resulted in no motion, some motion and motion as acceleration increased.

Qualitatively we found that initiation of motion occurs at or is very close to the maximum shear stress due to the combined effects of drag/lift and acceleration as introduced by Nielsen and Callaghan (2003). However, quantitatively their formulation does not lead to convincing discrimination between motion and no-motion. We expect this to be due to the assumption that the coefficients for drag/lift and acceleration in their formulation are taken equal. From literature and from plotting our data against the Keulegan-Carpenter number we expect that the coefficients strongly vary caused by flow separation effects.

To arrive at a more convincing discrimination between motion and no-motion we introduced a new fluid acceleration descriptor for nonlinear shoaling waves. The combination of this descriptor with a Reynolds number $\mathrm{Re}_{\mathrm{g}}$ clearly delineates the regions with particle motion and without particle motion and has the potential to serve as a descriptor of the incipient motion of coarse particles under nonlinear regular waves. The interesting property of the descriptor is that it simultaneously includes skewness and horizontal asymmetry, which is attractive in the formulation of future bed load transport formulations.

\section{Acknowledgements}

The experiments were conducted in a wave flume of the Hydraulics Laboratory of the Faculty of Civil Engineering and Geosciences of Delft University of Technology. Maarten Tromp 
conducted a first set of experiments as part of his MSc Thesis work, which laid the basis for the experiments conducted by Emanuele Terrile as part of his MSc thesis work (supported by the MSc EU Erasmus programme) reported here. The technical assistance in conducting these experiments by dr. Henry Fontijn is greatly appreciated. 


\section{Appendix A}

In order to judge the goodness-of-discrimination between motion and no-motion for the various formulations the following procedure was followed. A linear regression was made based on the no-motion (triangles) and some-motion results (circles). Subsequently the percentage of circles above the regression line and the percentage of triangles under that line was determined as well the averaged distance to the regression line for all circles (motion) above the line and the averaged distance of all triangles (no motion) under the line. This was done for the data presented in Figures A1, A2, and A3, which are the counterparts of figures 5, 7 and 10 . The below table presents the results.

\begin{tabular}{|l|l|}
\hline \multicolumn{2}{|l|}{ Formulation based on Nielsen and Callaghan $\theta$ vs $\operatorname{Re}_{g}$ (Fig A1 and Fig 5) } \\
\hline Triangles percentage under the line & $58.1 \%$ \\
\hline Average distance (for triangles under the line) to the separation line & 0.0039 \\
\hline Circles percentage above the line & $68.8 \%$ \\
\hline Average distance (for circles above the line) to the separation line & 0.0062 \\
\hline Formulation based on $a_{n l}$ vs $\operatorname{Re}_{g}$ (Fig A2 and Fig 7) & $72.6 \%$ \\
\hline Triangles percentage under the line & 0.0106 \\
\hline Average distance (for triangles under the line) to the separation line & $81.3 \%$ \\
\hline Circles percentage above the line & 0.0214 \\
\hline Average distance (for circles above the line) to the separation line & \multicolumn{2}{|l|}{} \\
\hline $\begin{array}{l}\text { Formulation based on Nielsen and Callaghan } \theta \text { with variable } C_{d} \text { and } C_{m} \text { vs } \operatorname{Re}_{g} \text { (Fig A1 and } \\
\text { Fig 5) }\end{array}$ \\
\hline Triangles percentage under the line & $62.9 \%$ \\
\hline Average distance (for triangles under the line) to the separation line & 0.0052 \\
\hline Circles percentage above the line & $53.1 \%$ \\
\hline Average distance (for circles above the line) to the separation line & 0.0171 \\
\hline
\end{tabular}




\section{Appendix B}

In the following table $1 \mathrm{~B}$, we report our results: the wave code number, the Reynolds-like number $R e_{g}$, the Reynolds number $R e, a_{n l}$ made dimensionless by the acceleration of gravity, the Shear stress from Nielsen and Callaghan $\theta$ by using $C_{d}=0.2 C_{m}$, the Shear stress from Nielsen's and Callaghan $\theta$ by using $C_{d}=0.2 C_{m}$. and the classes of movement ( $\mathrm{N}$ never, $\mathrm{S}$ sometime and A always.).

\begin{tabular}{|c|c|c|c|c|c|c|c|c|c|c|c|c|c|}
\hline Wave code & $\operatorname{Re}_{4}[-]$ & $\operatorname{Re}[-]$ & anitg [-] & \begin{tabular}{c|c|}
{$[-]$} \\
Nielsen's cq. \\
with $\mathrm{Cd}=0.2 \mathrm{Cm}$
\end{tabular} & $\begin{array}{c}\theta[-] \\
\text { Nielsen's eq. } \\
\text { with } C d=C \mathrm{~m}\end{array}$ & $\begin{array}{c}\text { Movement } \\
\text { class }\end{array}$ & Wave code & $\operatorname{Re}_{4}[-]$ & $\operatorname{Rie}[-]$ & anltg $[-]$ & \begin{tabular}{|c|}
$\theta[-]$ \\
Nielsen's ${ }^{\prime} \mathrm{eq}$. \\
with $\mathrm{Cd}=0.2 \mathrm{Cm}$
\end{tabular} & 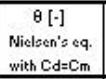 & $\begin{array}{c}\text { Movement } \\
\text { class }\end{array}$ \\
\hline$W 1$ & 919 & 1712 & 0.114 & 0.117 & 0.068 & $\mathrm{~N}$ & $W 60$ & 785 & 2063 & 0.140 & 0.120 & 0.081 & $\mathrm{~N}$ \\
\hline$w 2$ & 938 & 1778 & 0.127 & 0.128 & 0.073 & $\mathrm{~N}$ & W61 & 892 & 2407 & 0.155 & 0.084 & 0.065 & $\mathrm{~N}$ \\
\hline$w 3$ & 958 & 1918 & 0.129 & 0.156 & 0.089 & N & $W 62$ & 961 & 2512 & 0.174 & 0.134 & 0.086 & $\mathrm{~N}$ \\
\hline$W_{4}$ & 958 & 2002 & 0.127 & 0.175 & 0.097 & $\mathrm{~N}$ & $W 63$ & 1024 & 2658 & 0.194 & 0.116 & 0.085 & s \\
\hline W 5 & 938 & 2011 & 0.138 & 0.171 & 0.094 & N & $W 64$ & 1153 & 2570 & 0.169 & 0.107 & 0.081 & s \\
\hline W6 & 1221 & 2543 & 0.175 & 0.262 & 0.135 & N & $W 65$ & 825 & 1951 & 0.131 & 0.153 & 0.092 & s \\
\hline$w 7$ & 1181 & 2526 & 0.148 & 0.198 & 0.121 & N & $W 66$ & 709 & 1937 & 0.151 & 0.155 & 0.077 & $\mathrm{~s}$ \\
\hline W8 & 781 & 1681 & 0.112 & 0.109 & 0.076 & N & $W 67$ & 809 & 2131 & 0.154 & 0.115 & 0.087 & $\mathrm{~s}$ \\
\hline W9 & 954 & 2055 & 0.112 & 0.105 & 0.073 & N & W 68 & 957 & 2372 & 0.242 & 0.189 & 0.093 & s \\
\hline$W 10$ & 800 & 1742 & 0.107 & 0.114 & 0.082 & N & W 69 & 1005 & 2592 & 0.156 & 0.123 & 0.091 & $s$ \\
\hline$W 11$ & 977 & 2130 & 0.107 & 0.110 & 0.079 & $\mathrm{~N}$ & $W 70$ & 972 & 2552 & 0.176 & 0.153 & 0.098 & $\mathrm{~s}$ \\
\hline$W 12$ & 977 & 2191 & 0.125 & 0.128 & 0.087 & N & $W 71$ & 1153 & 2820 & 0.168 & 0.188 & 0.125 & s \\
\hline W/ 13 & 1009 & 2385 & 0.131 & 0.159 & 0.103 & $\mathrm{~N}$ & $W 72$ & 982 & 2583 & 0.180 & 0.236 & 0.145 & s \\
\hline W 14 & 819 & 2009 & 0.135 & 0.166 & 0.112 & $\mathrm{~N}$ & $W 73$ & 1073 & 2640 & 0.150 & 0.171 & 0.121 & s \\
\hline$W 15$ & 1001 & 2455 & 0.135 & 0.160 & 0.108 & $\mathrm{~N}$ & W 74 & 1135 & 2983 & 0.185 & 0.187 & 0.134 & $\mathrm{~s}$ \\
\hline W 16 & 774 & 2002 & 0.136 & 0.142 & 0.091 & $\mathrm{~N}$ & W 75 & 993 & 2587 & 0.140 & 0.253 & 0.155 & $\mathrm{~s}$ \\
\hline W 17 & 951 & 2336 & 0.130 & 0.093 & 0.063 & $\mathrm{~N}$ & $W 76$ & 804 & 2052 & 0.160 & 0.130 & 0.092 & s \\
\hline W 18 & 1040 & 2552 & 0.159 & 0.103 & 0.071 & $\mathrm{~N}$ & W 77 & 903 & 2442 & 0.196 & 0.164 & 0.102 & s \\
\hline$W 19$ & 910 & 2389 & 0.143 & 0.069 & 0.055 & $\mathrm{~N}$ & W 78 & 1187 & 3128 & 0.216 & 0.169 & 0.119 & s \\
\hline$W 20$ & 965 & 2429 & 0.142 & 0.063 & 0.050 & $\mathrm{~N}$ & W 79 & 714 & 1915 & 0.137 & 0.208 & 0.127 & s \\
\hline$W 21$ & 1041 & 2570 & 0.148 & 0.128 & 0.092 & $\mathrm{~N}$ & W 80 & 1109 & 2534 & 0.193 & 0.140 & 0.104 & s \\
\hline$W 22$ & 1094 & 2878 & 0.154 & 0.157 & 0.093 & $\mathrm{~N}$ & W 81 & 880 & 2254 & 0.173 & 0.137 & 0.103 & s \\
\hline$W 23$ & 1101 & 2970 & 0.167 & 0.153 & 0.090 & N & W 82 & 1093 & 2578 & 0.153 & 0.153 & 0.098 & s \\
\hline$W 24$ & 1153 & 3062 & 0.151 & 0.143 & 0.091 & $\mathrm{~N}$ & W 83 & 1069 & 2860 & 0.230 & 0.214 & 0.114 & s \\
\hline$W 25$ & 912 & 2350 & 0.131 & 0.109 & 0.076 & $\mathrm{~N}$ & W 84 & 705 & 1915 & 0.167 & 0.122 & 0.074 & $s$ \\
\hline$W 26$ & 1032 & 2627 & 0.147 & 0.122 & 0.090 & $\mathrm{~N}$ & $W 85$ & 851 & 2102 & 0.148 & 0.127 & 0.091 & s \\
\hline$W 27$ & 873 & 2341 & 0.137 & 0.120 & 0.073 & N & W 86 & 1120 & 2847 & $\begin{array}{l}0.167 \\
0.167\end{array}$ & 0.084 & 0.058 & s \\
\hline$W 28$ & 1120 & 2394 & 0.127 & 0.170 & 0.095 & $\mathrm{~N}$ & W 87 & 1036 & 2715 & 0.206 & 0.137 & 0.081 & s \\
\hline$W 29$ & 1163 & 2468 & 0.129 & 0.194 & 0.107 & N & W 88 & 961 & 2596 & 0.192 & 0.174 & 0.111 & s \\
\hline$W 30$ & 1023 & 2310 & 0.133 & 0.116 & 0.073 & $\mathrm{~N}$ & $W 89$ & 944 & 2102 & 0.169 & 0.116 & 0.087 & s \\
\hline$W 31$ & 951 & 2464 & 0.166 & 0.112 & 0.070 & $\mathrm{~N}$ & W 90 & 787 & 2081 & 0.190 & 0.154 & 0.099 & s \\
\hline W 32 & 779 & 1782 & 0.112 & 0.078 & 0.054 & $\mathrm{~N}$ & W 91 & 786 & 2056 & 0.174 & 0.145 & 0.093 & s \\
\hline$W 33$ & 762 & 1832 & 0.120 & 0.088 & 0.058 & $\mathrm{~N}$ & W 92 & 908 & 2074 & 0.193 & 0.113 & 0.086 & s \\
\hline$W 34$ & 1056 & 2640 & 0.148 & 0.135 & 0.093 & $\mathrm{~N}$ & W 93 & 937 & 2499 & 0.171 & 0.157 & 0.106 & s \\
\hline$W 35$ & 844 & 2149 & 0.147 & 0.123 & 0.090 & $\mathrm{~N}$ & W 94 & 1011 & 2640 & 0.208 & 0.262 & 0.136 & s \\
\hline$W 36$ & 1070 & 2556 & 0.139 & 0.145 & 0.083 & $\mathrm{~N}$ & W 95 & 997 & 2284 & 0.245 & 0.172 & 0.106 & A \\
\hline W 37 & 951 & 2006 & 0.133 & 0.073 & 0.051 & N & $W 96$ & 745 & 1955 & 0.143 & 0.113 & 0.070 & A \\
\hline W 38 & 965 & 2226 & 0.138 & 0.100 & 0.063 & $\mathrm{~N}$ & W 97 & 1023 & 2486 & 0.142 & 0.238 & 0.131 & A \\
\hline$W 39$ & 1101 & 2675 & 0.155 & 0.183 & 0.113 & $\mathrm{~N}$ & W 98 & 1181 & 2781 & 0.185 & 0.325 & 0.179 & A \\
\hline$W 40$ & 762 & 1912 & 0.127 & 0.101 & 0.059 & $\mathrm{~N}$ & W 99 & 739 & 1998 & 0.196 & 0.172 & 0.107 & A \\
\hline W 41 & 1181 & 2763 & 0.171 & 0.251 & 0.131 & N & $W 100$ & 838 & 2174 & 0.194 & 0.145 & 0.105 & A \\
\hline$W^{4}$ & 778 & 1912 & 0.130 & 0.084 & 0.049 & $\mathrm{~N}$ & $W 101$ & 816 & 1868 & 0.245 & 0.160 & 0.101 & A \\
\hline$W 43$ & 1202 & 2332 & 0.144 & 0.177 & 0.106 & N & W 102 & 860 & 1966 & 0.221 & 0.169 & 0.108 & $\mathrm{~A}$ \\
\hline W 44 & 1211 & 2416 & 0.154 & 0.227 & 0.122 & $\mathrm{~N}$ & $W 103$ & 1206 & 3318 & 0.242 & 0.193 & 0.110 & A \\
\hline W 45 & 823 & 2120 & 0.156 & 0.129 & 0.096 & $\mathrm{~N}$ & W 104 & 1051 & 2402 & 0.221 & 0.161 & 0.102 & A \\
\hline$W 46$ & 867 & 2367 & 0.151 & 0.153 & 0.081 & $\mathrm{~N}$ & W 105 & 1071 & 2583 & 0.151 & 0.145 & 0.091 & A \\
\hline$W 47$ & 1271 & 3256 & 0.148 & 0.094 & 0.076 & $\mathrm{~N}$ & W 106 & 864 & 2160 & 0.148 & 0.134 & 0.093 & A \\
\hline$W 48$ & 988 & 2605 & 0.154 & 0.113 & 0.086 & $\mathrm{~N}$ & W 107 & 1266 & 3454 & 0.168 & 0.100 & 0.068 & A \\
\hline$W 49$ & 983 & 2508 & 0.160 & 0.124 & 0.088 & N & W 108 & 1202 & 2552 & 0.186 & 0.258 & 0.148 & A \\
\hline W 50 & 800 & 1793 & 0.125 & 0.115 & 0.071 & $\mathrm{~N}$ & $W 109$ & 730 & 1969 & 0.155 & 0.291 & 0.158 & A \\
\hline W 51 & 1243 & 3282 & 0.176 & 0.101 & 0.080 & N & $W 110$ & 1051 & 2772 & 0.203 & 0.288 & 0.151 & $\mathrm{~A}$ \\
\hline W 52 & 746 & 1922 & 0.131 & 0.096 & 0.064 & $\mathrm{~N}$ & $W 111$ & 767 & 2045 & 0.171 & 0.189 & 0.120 & A \\
\hline W 53 & 1143 & 2864 & 0.168 & 0.077 & 0.060 & N & $W^{112}$ & 795 & 2088 & 0.176 & 0.184 & 0.111 & A \\
\hline W 54 & 1047 & 2715 & 0.162 & 0.099 & 0.076 & N & $W 113$ & 786 & 2124 & 0.192 & 0.208 & 0.125 & A \\
\hline W5 55 & 790 & 1987 & 0.142 & 0.067 & 0.050 & N & W 114 & 1092 & 2904 & 0.233 & 0.294 & 0.125 & A \\
\hline W 56 & 1075 & 2754 & 0.173 & 0.110 & 0.083 & N & $W 115$ & 1235 & 3388 & 0.202 & 0.159 & 0.099 & A \\
\hline W 57 & 962 & 2543 & 0.190 & 0.142 & 0.091 & N & $W 116$ & 1280 & 3516 & 0.255 & 0.188 & 0.111 & A \\
\hline W 58 & 1031 & 2543 & 0.144 & 0.154 & 0.097 & N & W 117 & 1269 & 3480 & 0.241 & 0.179 & 0.097 & $\hat{A}$ \\
\hline$W 59$ & 861 & 2341 & 0.167 & 0.085 & 0.065 & $\mathrm{~N}$ & & & & & & & \\
\hline
\end{tabular}

Table 1B: Data results 


\section{References}

Bagnold, R A (1963): Beach and nearshore processes: Part I, Mechanics of marine sedimentation. In N M Hill Ed The Sea Vo13: The earth beneath the sea, pp 507-553.

Drake, T.G., and Calantoni, J. (2001), Discrete particle mode for sheet flow sediment transport in the nearshore. Journal of Geophysiscal Res. Oceans, Vol. 106, No C9 pages 19.85919.868 .

Elgar, S., and Guza, R.T. (1985), Observation of bispectra of shoaling surface gravity waves. Journal of Fluid Mechanics. Vol. 167, pages 425-448.

Fredsøe, J., and Deigaard, R. (1992), Mechanics of coastal sediment transport. Advanced Series on Ocean Engineering, Vol. 2, World Scientific.

Hallermeier, R J (1980): Sand motion initiation by water waves; two asymptotes. Proc A S C E, Vo1106, no WW6, pp 299-318

Hoefel, F., and Elgar, S. (2003), Wave-induced sediment transport and sand bar migration. Science, Vol. 299, pages 1885-1887.

Jonsson, I.G. (1966), Wave boundary Layers and friction factor. Proc.10th ICCE Tokyo, ASCE, New York.

Jonsson, I.G., and Svendsen, I.A. (1976), Hydrodynamics of coastal regions. Technical University of Denmark.

Keulegan, G.H. and L.H. Carpenter (1958), Forces on cylinders and plates in an oscillating fluid, J. Res. Nat. Bur. Stand., Vol. 60, No. 5.

King, D B, Jr (1991): Studies on oscillatory flow bedload sediment transport. PhD thesis U C San Diego (Scripps).

Madsen, O S (1974): Stability of a sand bed under breaking waves. Proc $14^{\text {th }}$ Int Conf Coastal Eng, Copenhagen, AS C E, pp 776-794.

Nielsen, P. (1992), Coastal bottom boundary layers and sediment transport. Advanced Series on Ocean Engineering, Vol. 4, World Scientific.

Nielsen, P. (2002), Shear stress and sediment transport calculations for swash zone modelling. Coastal Engineering, Vol. 45, pages 53-67.

Nielsen, P., and Callaghan, D.P. (2003), Shear stress and sediment transport calculations for sheet flow under waves. Coastal Engineering, Vol. 47, pages 347-354.

Ribberink, J S, C M Dohmen-Janssen, DM Hanes, S R McLean \& C Vincent (2000): Near bed sand transport mechanisms under waves. Proc 21h Int Con/Coastal Eng, Sydney, AS C E, New York, pp3263-3276.

Sarpkaya, T. (1976), Vortex shedding and resistance in harmonic flow about smooth and rough circular cylinders at high Reynolds numbers, Rep. NPS-59SL76021, U.S. Naval Postgraduate School.

Sleath, J FA (1994): Bedload transport in oscillatory flow. In Belogey, Rajaona \& Sleath Ed: Sediment transport mechanisms in coastal environments and rivers (Proc Euromech 310), World Scientific.

Sleath, J FA (1999): Conditions for plug formation in oscillatory flow. Cont Shelf Res, Vo119, pp 1643-1664.

Stive, M.J.F., and Reniers, A.J.H.M. (2003), Sandbars in motion. Science, Vol. 299, pages $1855-1856$.

Terrile, E. (2004), The threshold of motion of coarse sediment particles by regular nonbreaking waves. Master's Thesis, University of Genoa and Delft University of Technology (www.hydraulicengineering.tudelft.nl).

Tromp, M. (2004), The influence that fluid accelerations have on threshold of motion. Master's Thesis, Delft University of Technology (www.hydraulicengineering.tudelft.nl).

Zala-Flores, N \& J FA Sleath (1998): Mobile layer in oscillatory sheet flow. J Geophys Res Vol 103 No C6, pp 12783-12793. 


\section{List of figures}

Figure 1: Flume dimensions and location of measurement instruments along the slope (horizontal dimensions in meters).

Figure 2: Comparison of Hallermeier's equation (1) with our test data.

Figure 3: Time series of near-bed velocity (red line) and acceleration (blue line).

Figure 4: Velocity and acceleration terms of the stability parameter and the stability parameter $\theta_{a}$ for a wave with period $T=3 \mathrm{~s}$ and height $H=0.15 \mathrm{~m}$.

Figure 5: Shields like presentation of Nielsen's max shear stress vs Re (based on $u_{r m s}$ )

Figure 6: Shields like presentation of Nielsen's max shear stress vs Re (based on $u_{\text {max }}$ )

Figure 7: Threshold of motion line (A) never, (o) sometimes, (x) always, as a function of $a_{n} / g$ and $R_{g}$.

Figure 8: Threshold of motion line ( $\Delta$ ) never, (o) sometimes, ( $x$ ) always, as a function of $a_{n} / g$ and $u_{r m s}^{2} / g D_{50}$.

Figure 9: Threshold of motion line ( $\Delta$ ) never, (o) sometimes, ( $x$ ) always, as a function of $a_{n l}$ $D_{50} / g^{2} T^{2}$ and the Keulegan-Carpenter number $u_{r m s} T / D_{50}$.

Figure 10: Shields like presentation of Nielsen 'max shear stress (based on $C_{d} \approx 0.2 C_{m}$ ) vs $R e_{g}$.

Figure 11: Time series of near-bed velocity (solid line) and acceleration (dashdot line) from simulated waveforms having $\Phi=0$.

Figure 12: Ratio between $\left\langle u^{3}\right\rangle$ and $a^{3}{ }_{n l}$ for different simulated waveforms having $\Phi=0, \pi / 8$, $\pi / 4,3 \pi / 8$ and $\pi / 2, T=6 s$ and different velocity amplitudes $c=0.1,0.5,1.0,1.5$ and 2.0 $\mathrm{m} / \mathrm{s}$.

Figure 13: Time series of near-bed velocity (solid line) and acceleration (dashdot line) from simulated waveforms having $\Phi=\pi / 2$.

Figure 14: Relation between $a_{n l}$ and $a_{\text {spike }}$ from simulated waveforms having $\Phi=0, \pi / 4$, and $\pi / 2, T=6 \mathrm{~s}$ and different velocity amplitudes $c=0.1,0.5,1.0,1.5$ and $2.0 \mathrm{~m} / \mathrm{s}$.

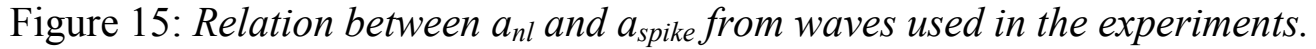

Figure A1: Nielsen and Callaghan $\theta$ vs $R_{g}$.

Figure A2: $a_{n} / g$ vs $R e_{g}$.

Figure A3: Nielsen and Callaghan $\theta$ (based on $C_{d} \approx 0.2 C_{m}$ ) vs $R e_{g}$. 


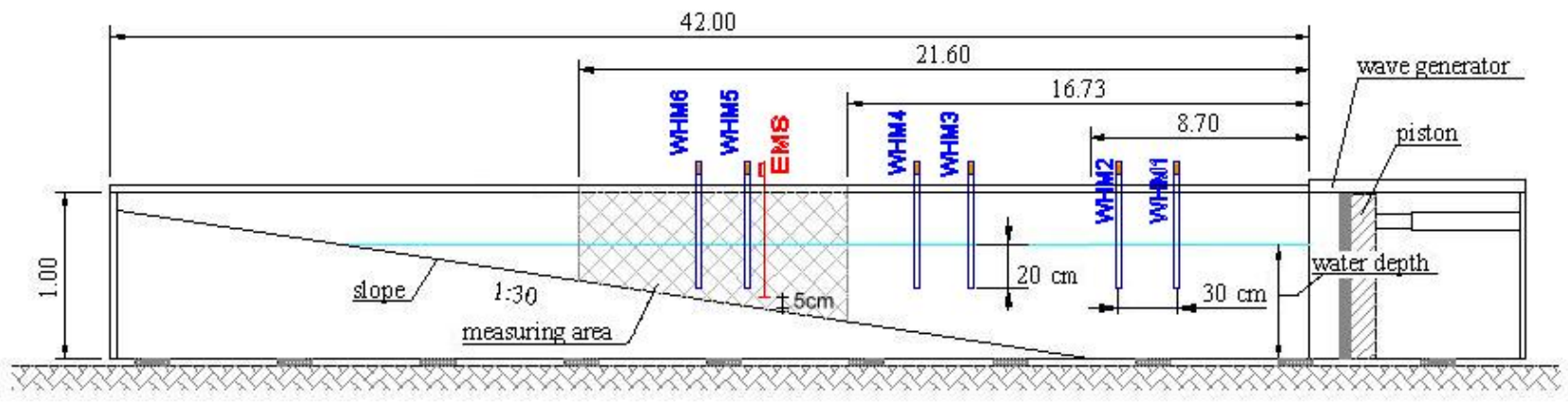

Figure 1

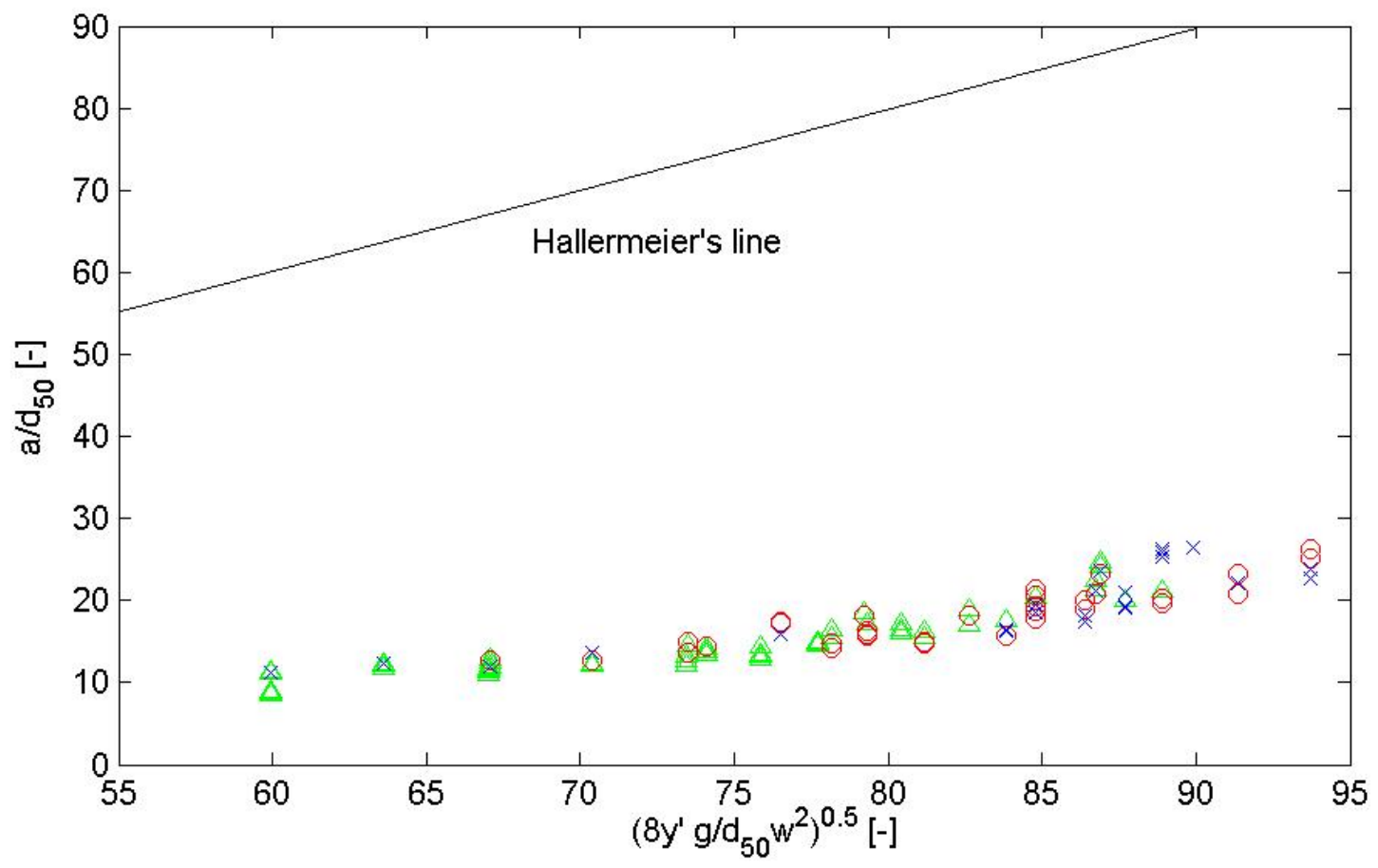

Figure 2 


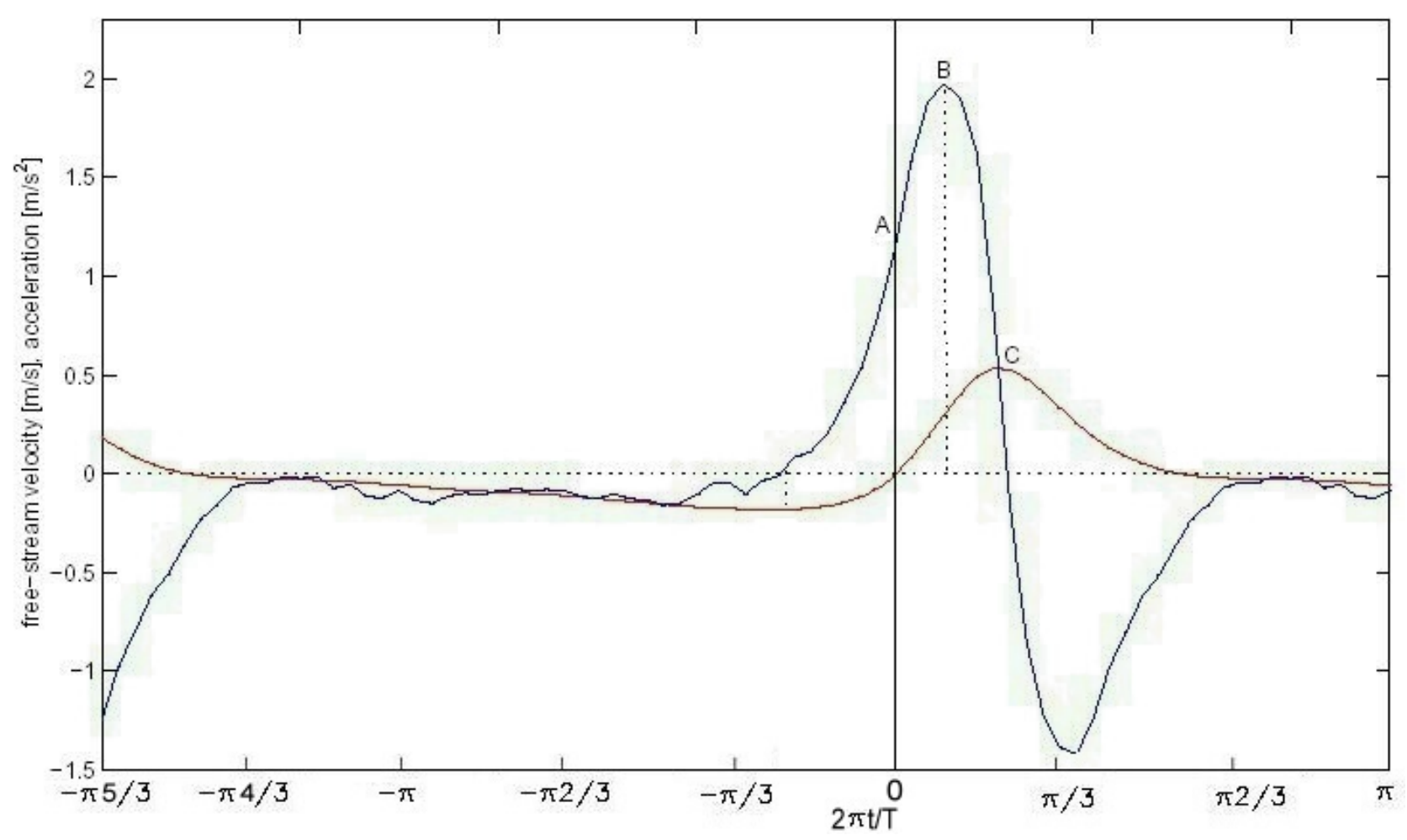

Figure 3

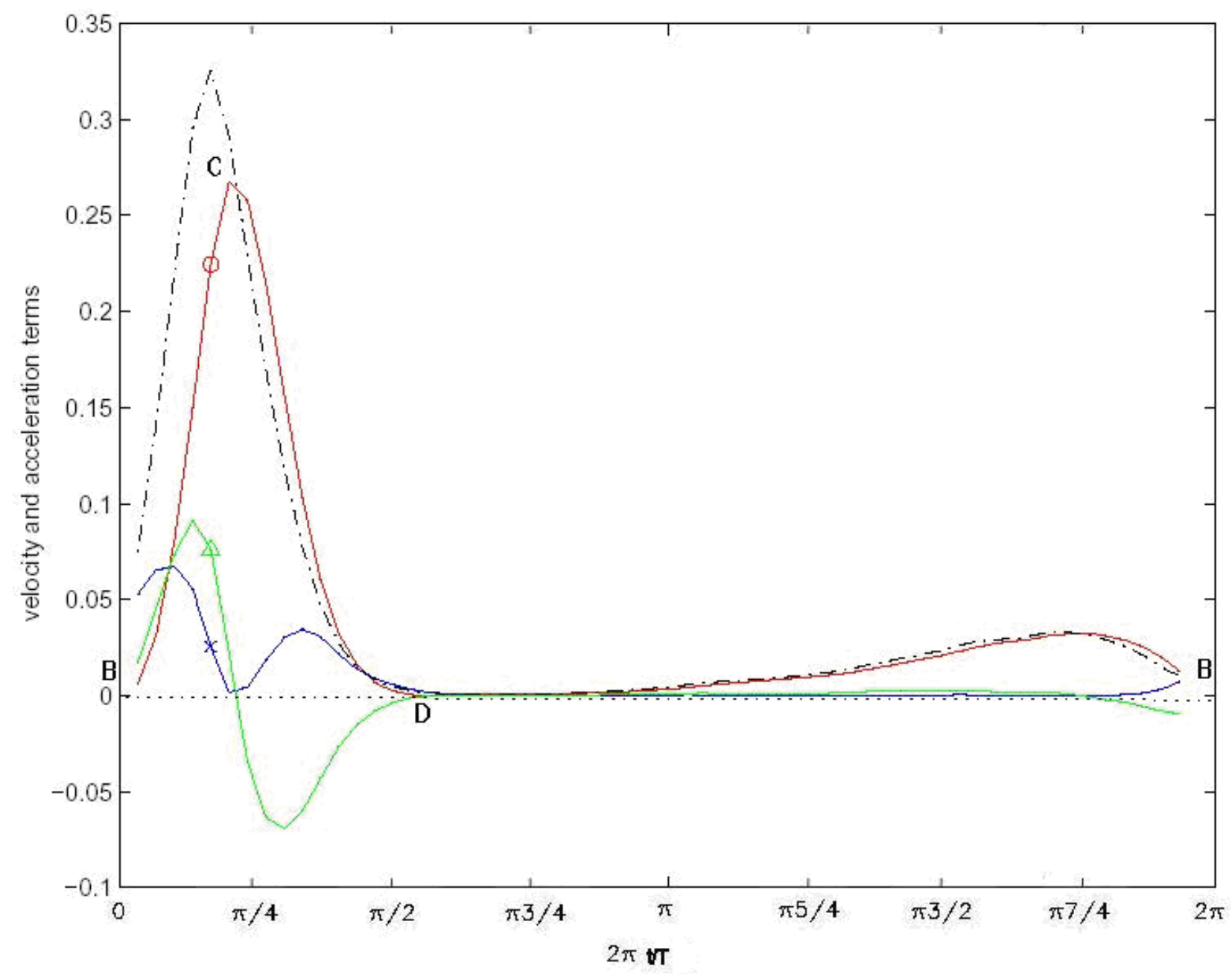

Page 15 of 24 
Figure 4

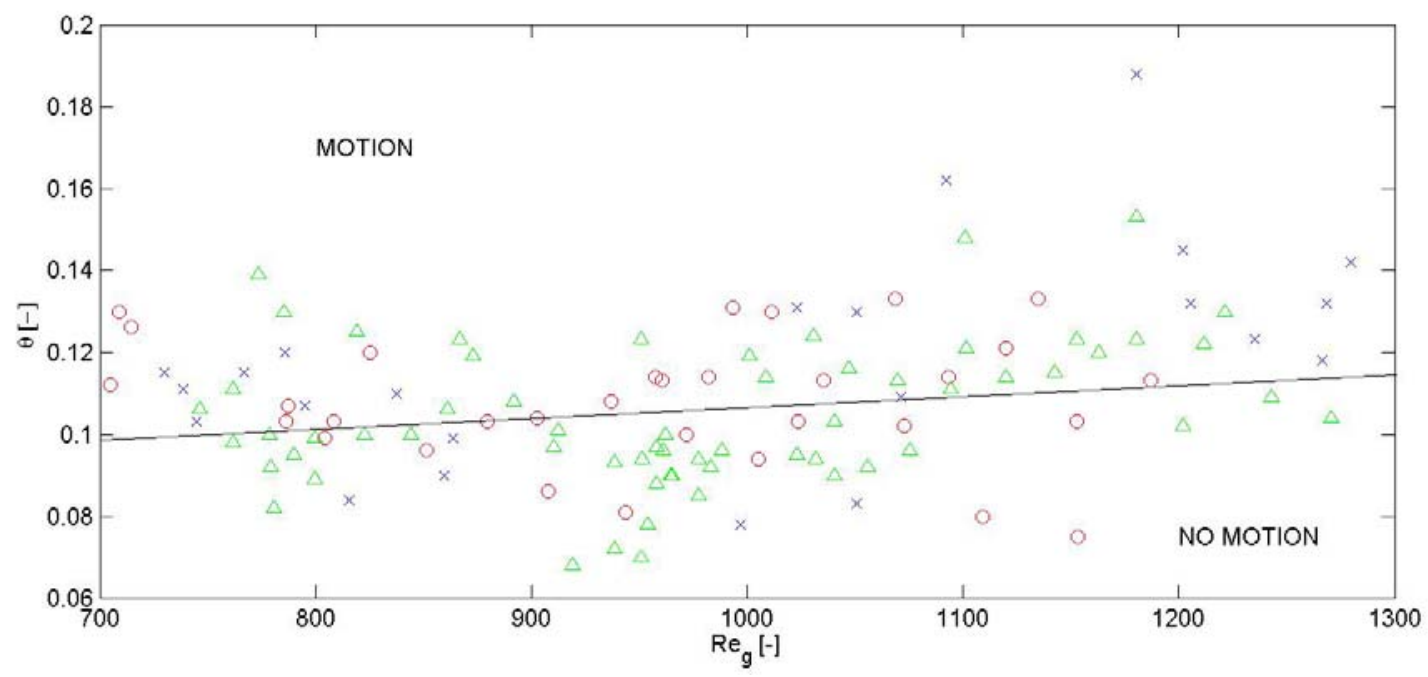

Figure 5

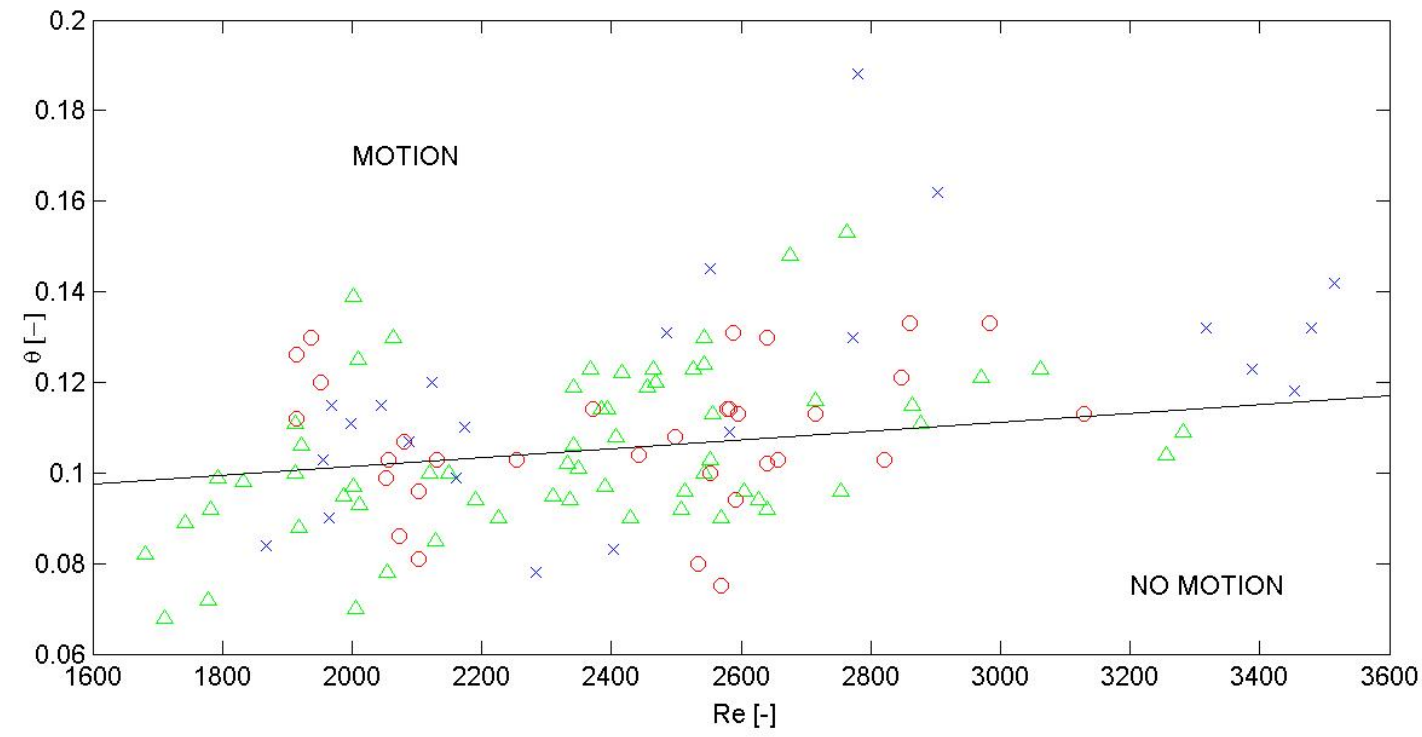

Figure 6 


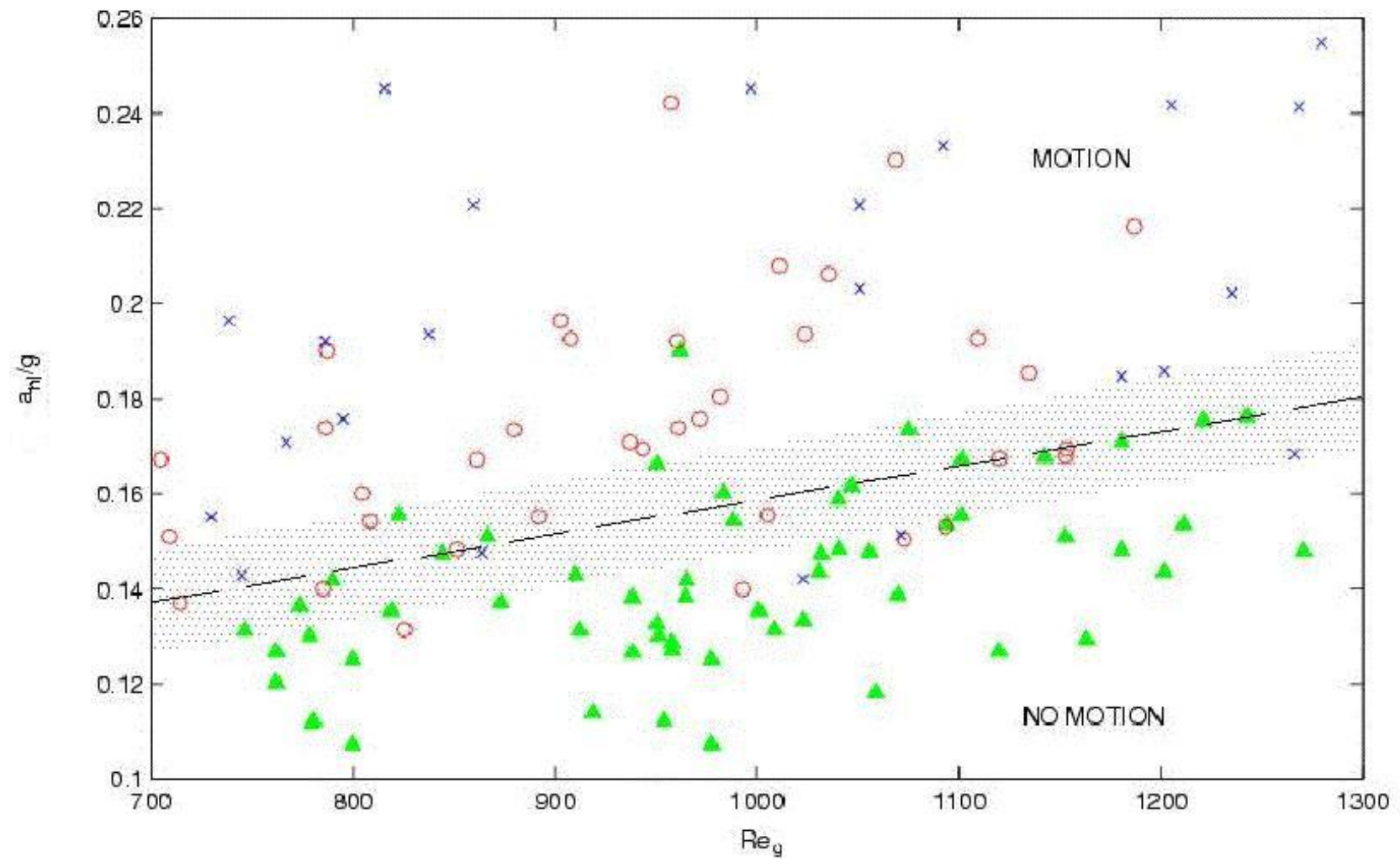

Figure 7

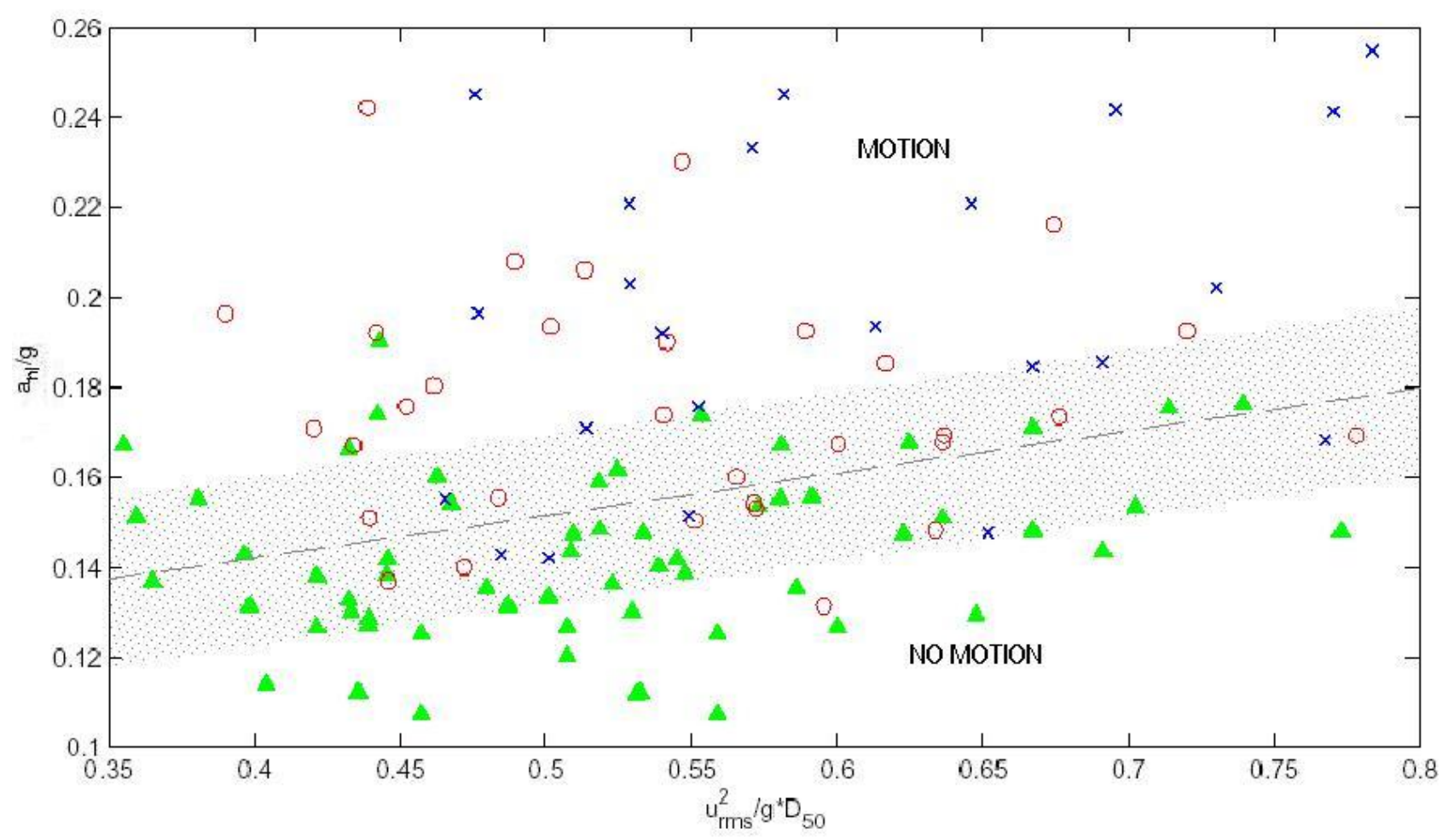

Figure 8 


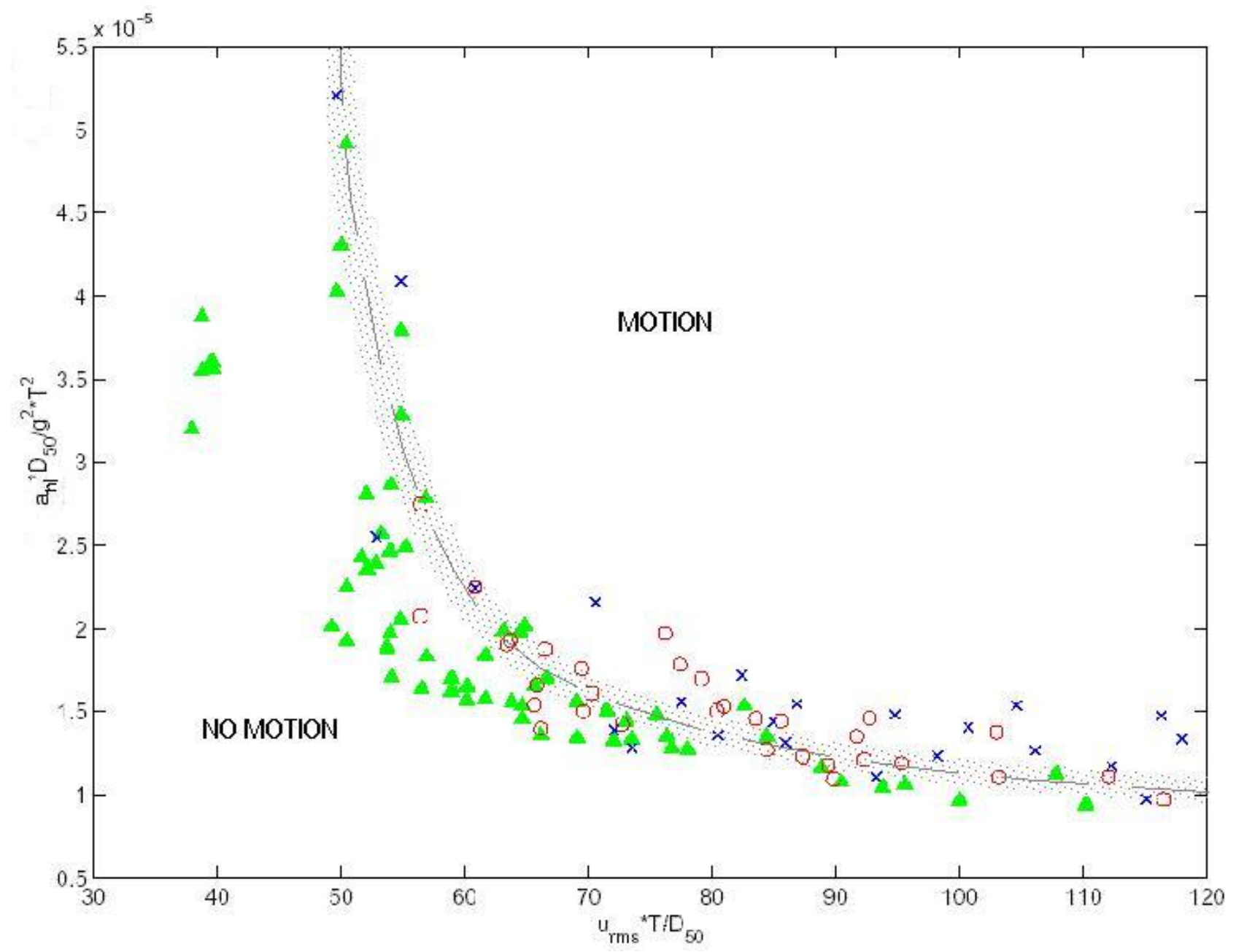

Figure 9

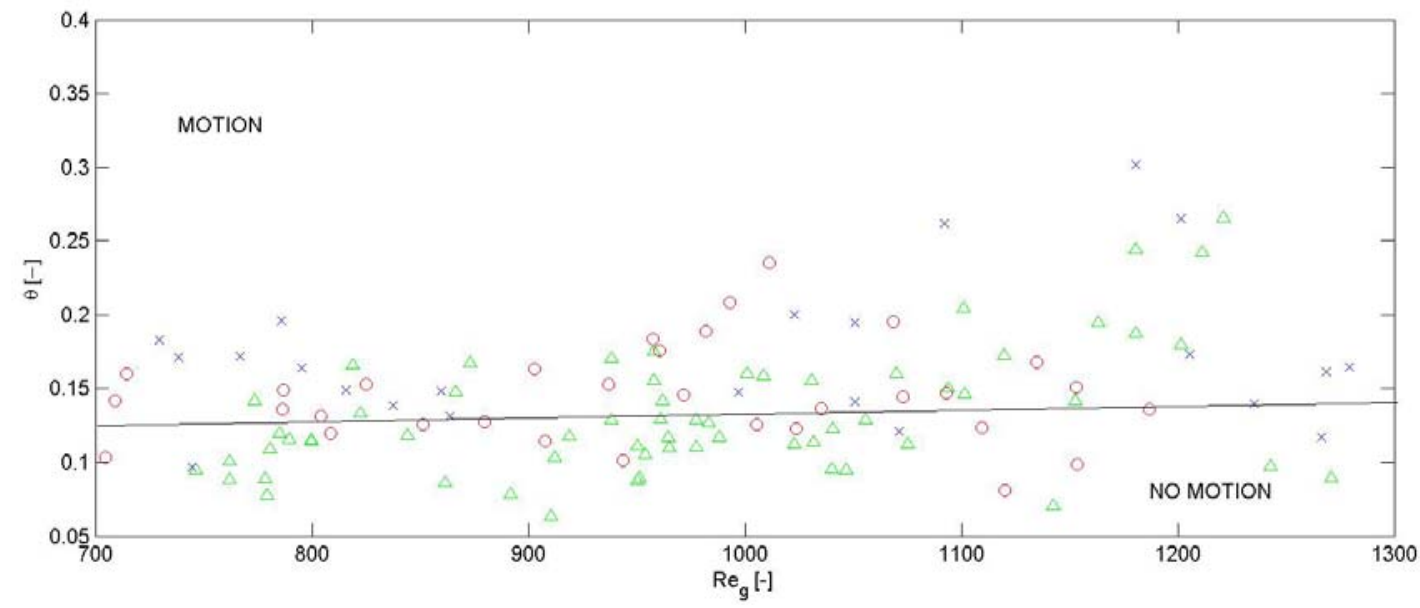

Figure 10 


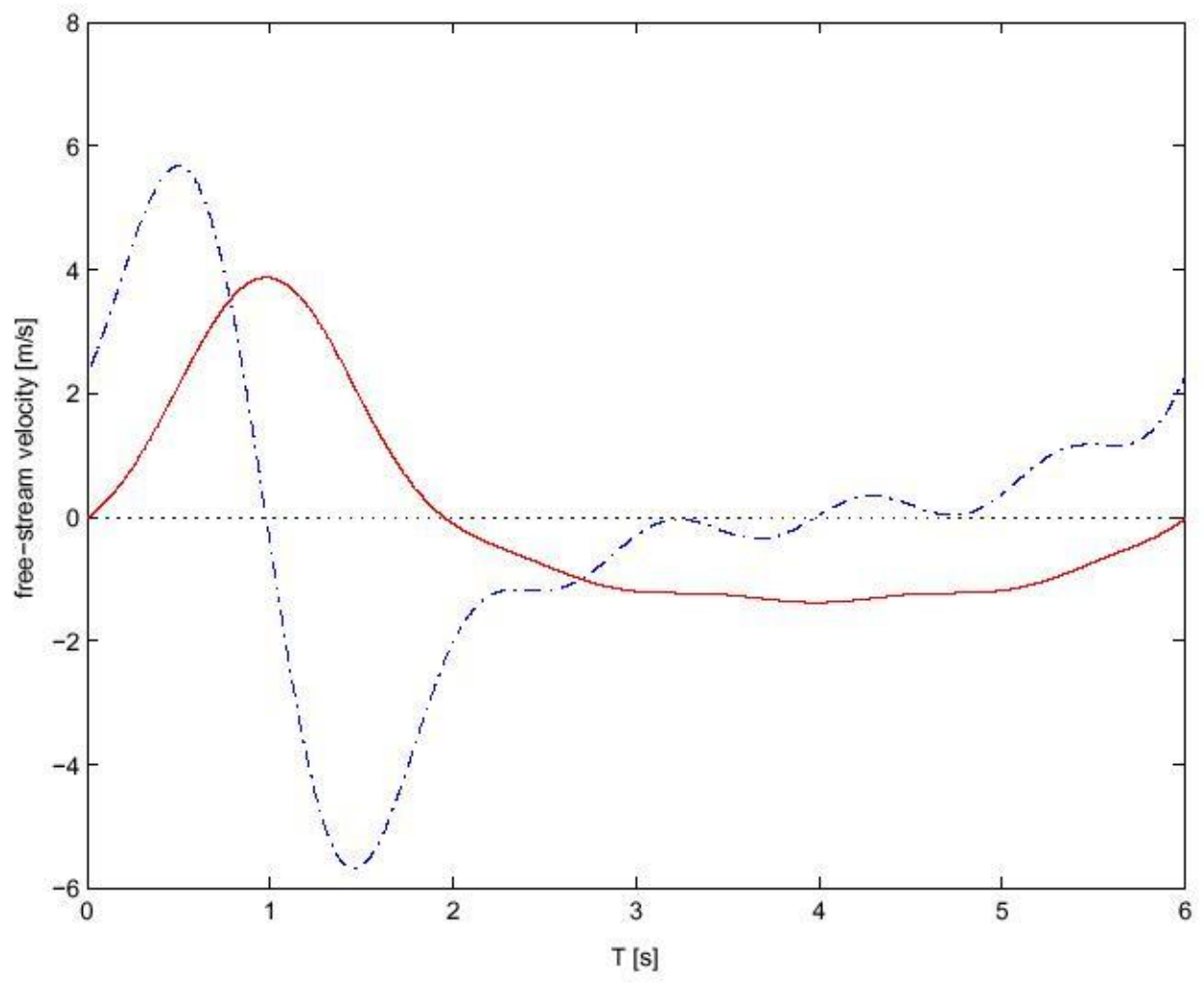

Figure 11 


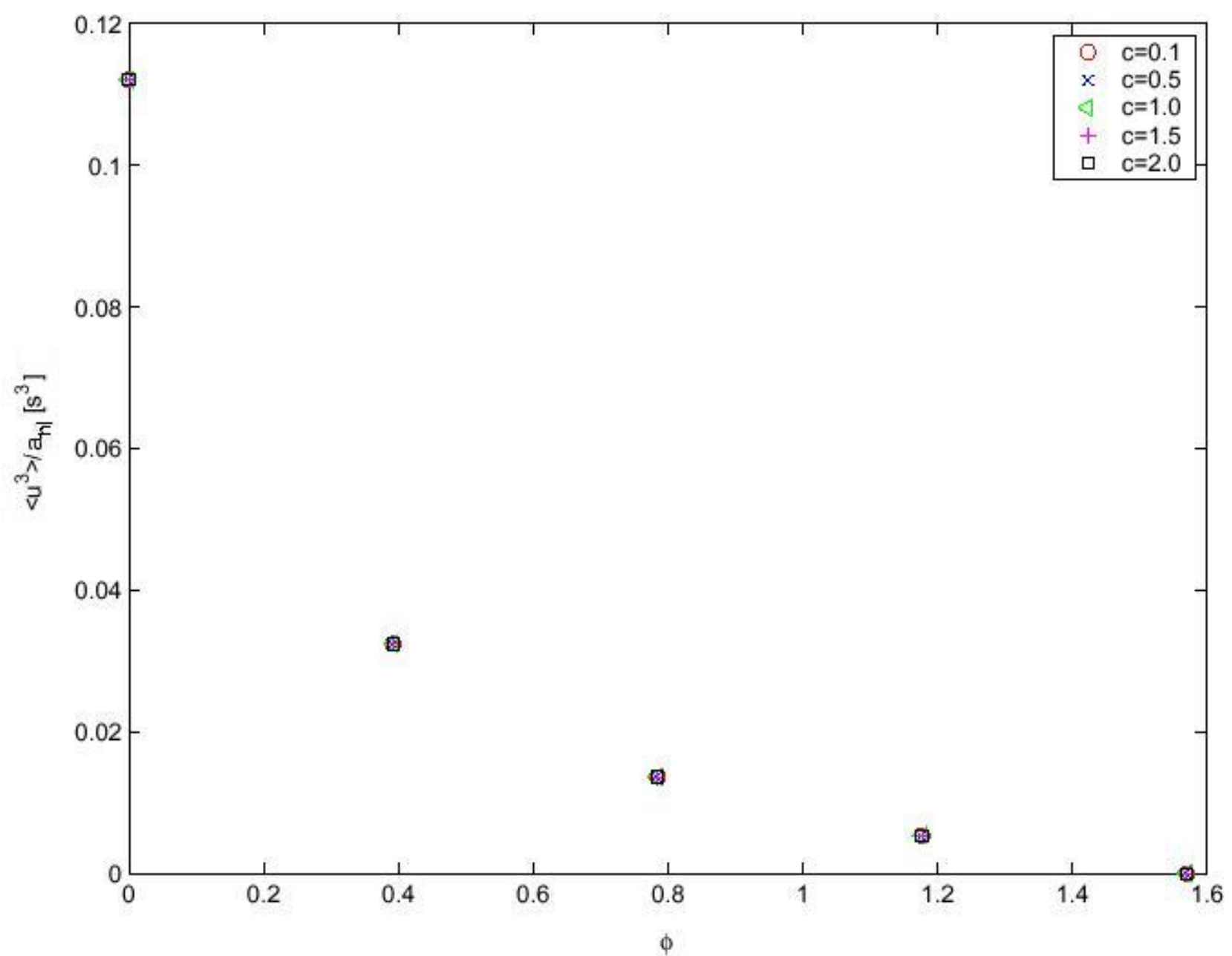

Figure 12 


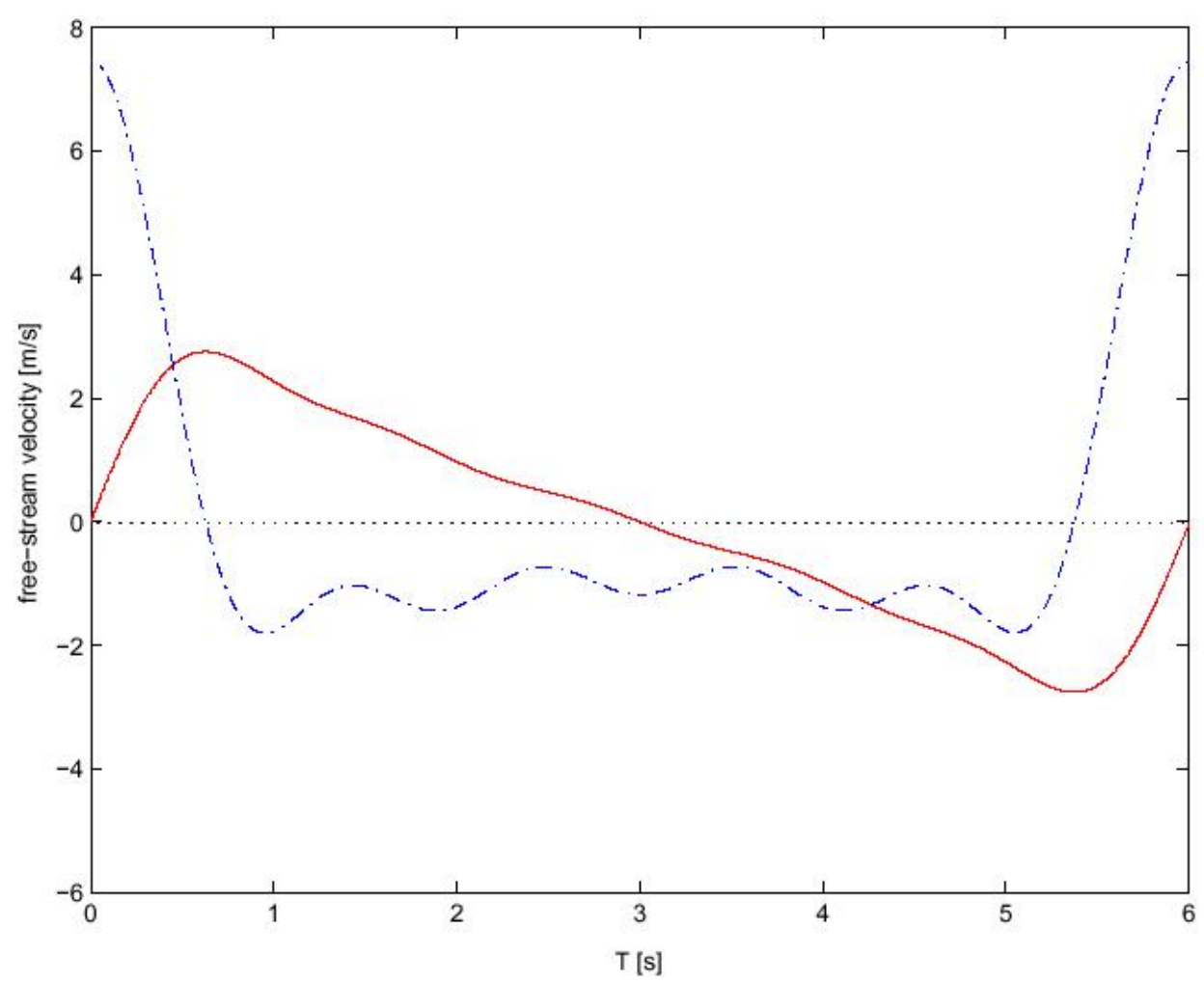

Figure 13 


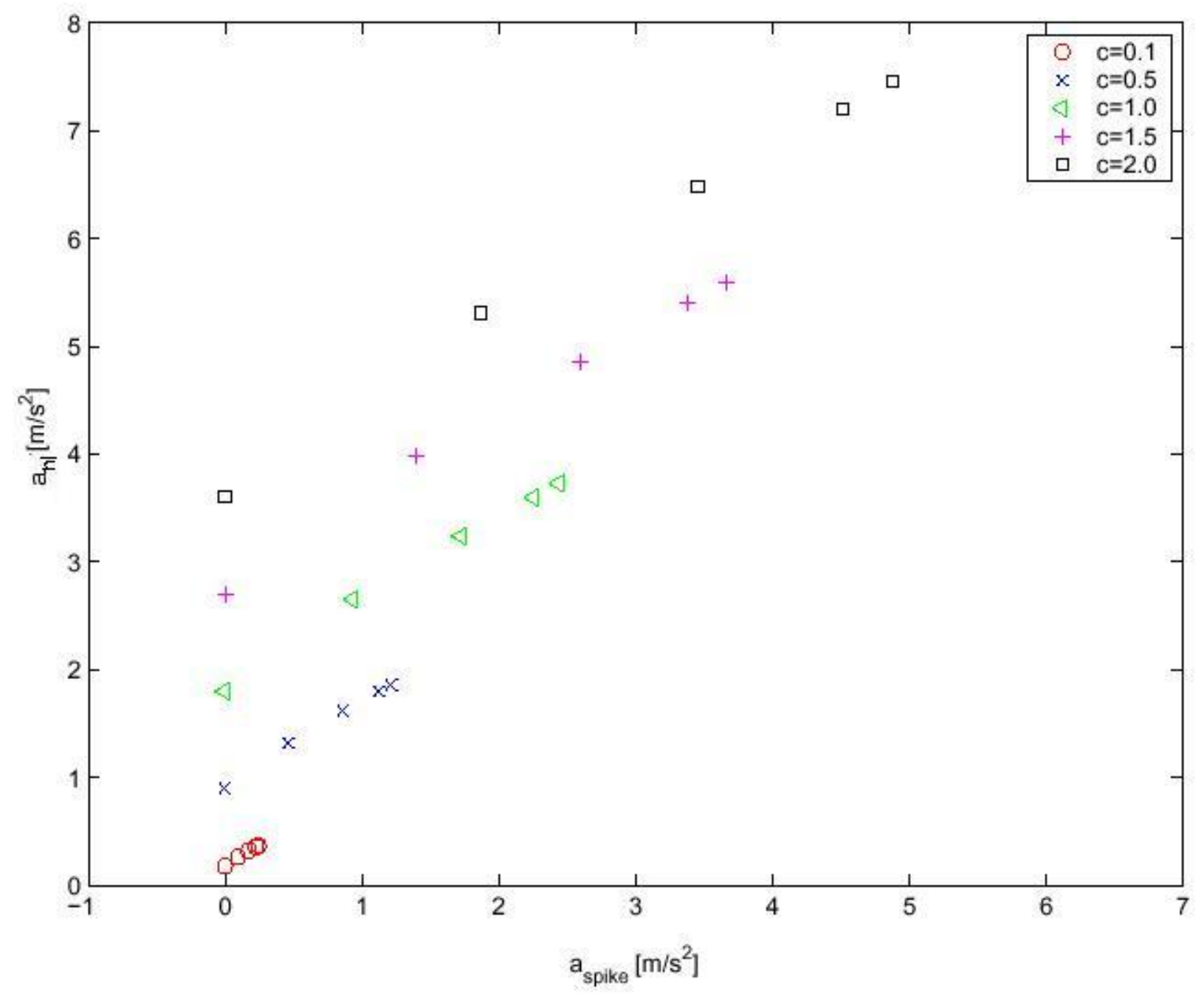

Figure 14 


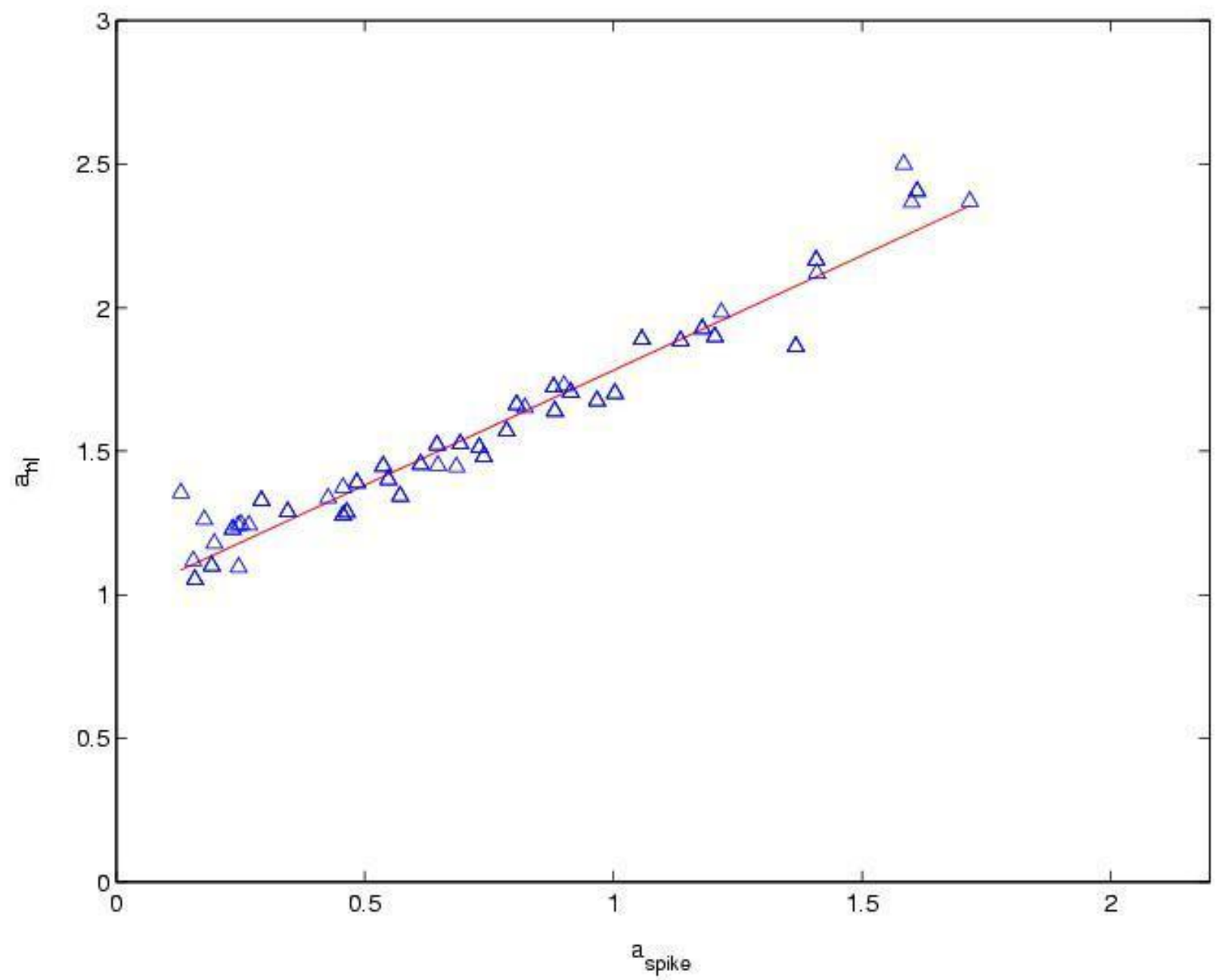

Figure 15

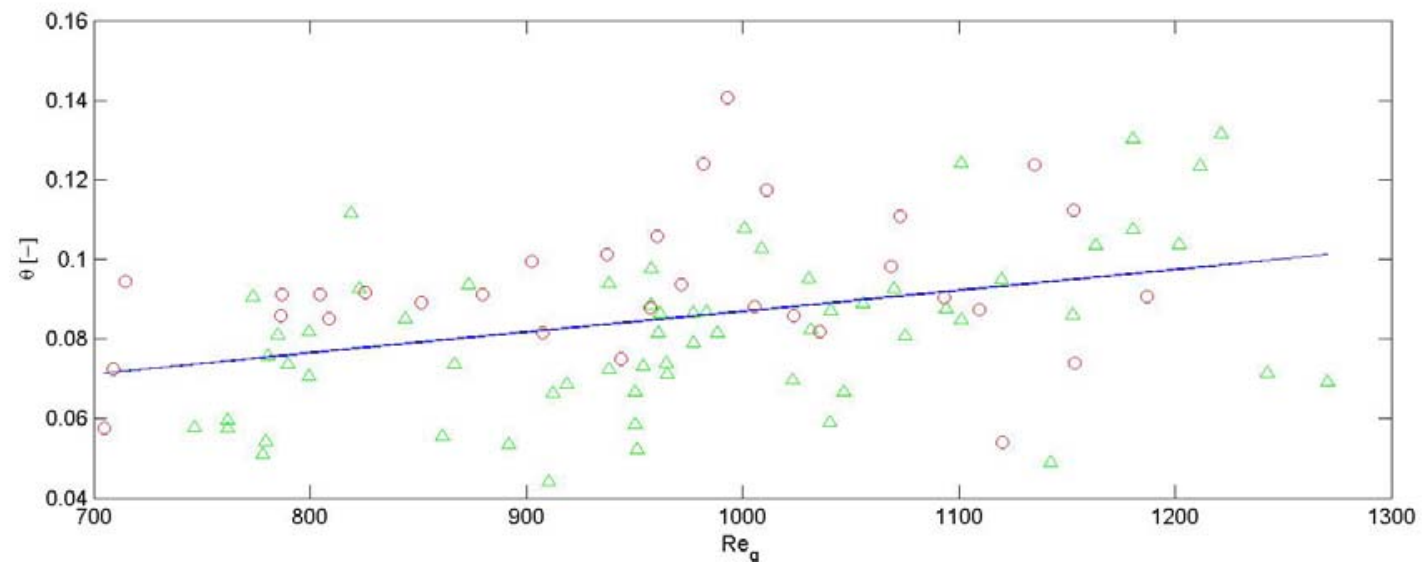

Figure A1 


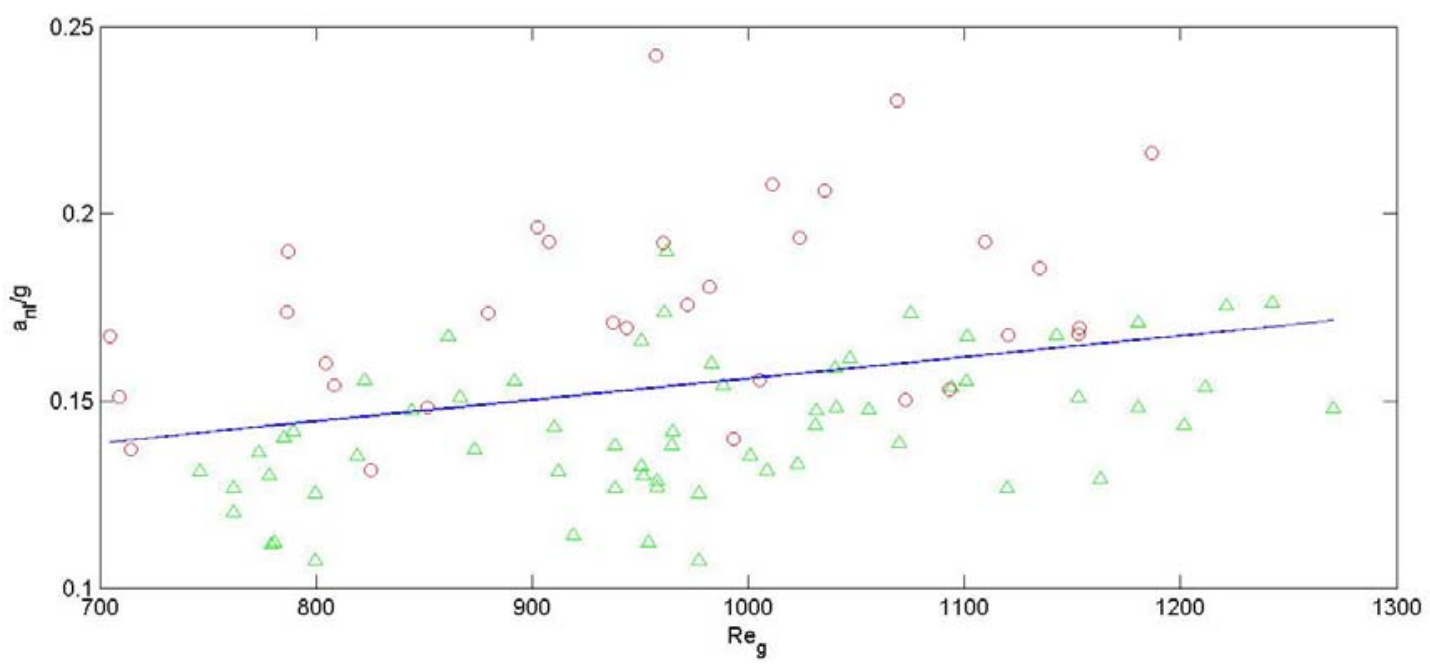

Figure A2

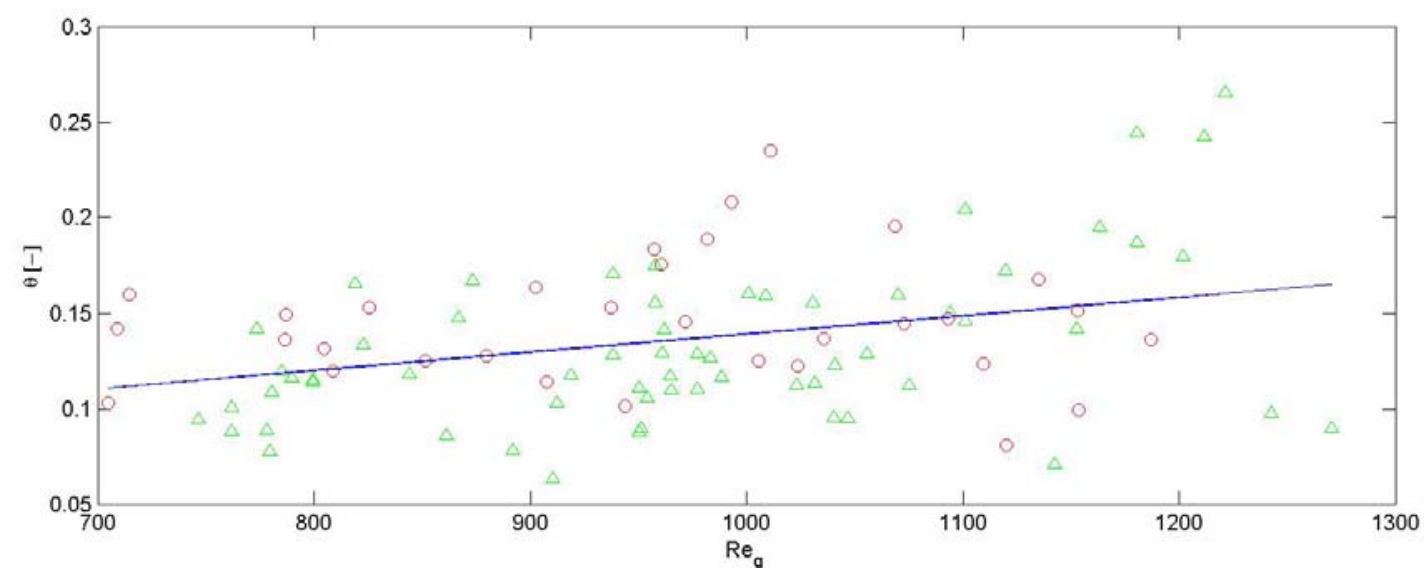

Figure A3 